\title{
SÍNDROME NEUROLÉPTICA MALIGNA
}

\section{RELATO DE CASO COM RECORRÊNCIA ASSOCIADA AO USO DE OLANZAPINA}

\author{
RICARDO A. HANEL, MARCOS C. SANDMANN, MARTINA KRANICH, PAULO R. M. DE BITTENCOURT
}

\begin{abstract}
RESUMO - A síndrome neuroléptica maligna (SNM) consiste em reação idiossincrática a neurolépticos, provavelmente relacionada a bloqueio dos receptores dopaminérgicos nos gânglios da base, sendo por isso também conhecida como síndrome da deficiência aguda de dopamina.A SNM é caracterizada por hiperpirexia, alteração do nível de consciência, hipertonia, disfunção autonômica e insuficiência respiratória, podendo ainda ser encontrados rabdomiólise e leucocitose. $\mathrm{O}$ haloperidol é a droga mais frequentemente associada à síndrome. Relatamos o caso de um paciente de 30 anos que apresentou SNM em duas ocasiões diferentes, a primeira delas relacionada ao uso de haloperidol e clorpromazina e a segunda relacionada ao uso de olanzapina, fato este sem menção anterior na literatura indexada.
\end{abstract}

PALAVRAS-CHAVE: síndrome neuroléptica maligna, haloperidol, clorpromazina, olanzapina, dantrolene, bromocriptina.

\section{Neuroleptic malignant syndrome: case report of a recurrence related to olanzapine}

ABSTRACT - The neuroleptic malignant syndrome (NMS) consists in an idiosyncratic reaction to neuroleptic drugs, probably related to a blockage of dopamine receptors in basal ganglia. Research criteria for diagnosing NMS from DSM-IV require severe rigidity and fever accompanied by 2 of 10 minor features including diaphoresis, dysphagia, tremor, incontinence, altered mentation, mutism, tachycardia, elevated or labile blood pressure, leukocytosis and elevation of creatine phosphokinase. From a clinical point of view, the NMS may range a large spectrum of presentations. Haloperidol is the most frequent drug associated with this syndrome. We report the case of a 30 year-old man who developed NMS at two different occasions, the first related to haloperidol and chlorpromazine and the second related to olanzapine, to our knowledge without previous mention in the indexed literature.

KEY WORDS: neuroleptic malignant syndrome, haloperidol. chlorpromazine, olanzapine, dantrolene, bromocriptine.

A síndrome neuroléptica maligna (SNM), inicialmente conhecida como rara complicação do uso dos antipsicóticos, é de tal maneira frequente, que qualquer médico neurologista, psiquiatra ou internista, trabalhando em um hospital geral, deparar-se-á com alguns casos no decorrer de poucos anos. Descrita pela primeira vez na literatura francesa em 1960 por Delay e col. ${ }^{1}$ e na literatura inglesa em 1968 por Delay e Deniker ${ }^{2}$ consiste de reação idiossincrática a agentes neurolépticos, provavelmente relacionada a bloqueio dos receptores dopaminérgicos nos gânglios da base, sendo por isso também conhecida como síndrome da deficiência aguda de dopamina ${ }^{3}$. Embora não exista definição universalmente aceita para ela, a SNM pode ser caracterizada por: hiperpirexia, alteração

Serviço de Neurologia e Neurofisiologia do Hospital Nossa Senhora das Graças, Curitiba PR. Aceite: 24agosto-1998.

Dr. Ricardo A. Hanel - Rua Alcides Munhoz, 433 - 80810-990 Curitiba PR - Brasil. FAX: 0413354549. E-mail : rhanel@hotmail.com 
do nível de consciência, hipertonia, disfunção autonômica e insuficiência respiratória, podendo ainda ser encontrados rabdomiólise e leucocitose ${ }^{4}$. Os principais agentes implicados na gênese da SNM são o haloperidol e a clorpromazina, havendo uma série de outras drogas e condições clínicas também associadas à síndrome.

O presente relato descreve o caso de um paciente de 30 anos que apresentou SNM em duas ocasiões diferentes, a primeira delas relacionada ao uso de haloperidol e clorpromazina e a segunda relacionada ao uso de olanzapina, fato este sem menção anterior na literatura indexada.

\section{RELATO DO CASO}

Este homem de 30 anos foi trazido ao ambulatório por dificuldade em acordar. Um familiar referiu que aproximadamente 15 dias antes, o paciente iniciou com idéias persecutórias; 2 dias antes da admissão foi iniciado tratamento com haloperidol $1 \mathrm{mg}$ ao dia. Após início da medicação, o familiar referiu aumento da agitação, sendo orientado a aumentar a dose para $5 \mathrm{mg}$ ao dia, associando-se clorpromazina $50 \mathrm{mg}$ ao dia. Na noite anterior à admissão, o paciente apresentou episódio de queda ao solo e movimentos involuntários dos membros com duração de cerca de 3 minutos. O psiquiatra orientou medicá-lo com diazepam $10 \mathrm{mg}$ via oral, após o que o paciente não mais acordou, sendo trazido na manhã seguinte ao hospital. Não havia história de episódios anteriores. Familiares negam história de uso de drogas. O paciente era portador do vírus da hepatite C (HCV) por transmissão vertical: mãe e irmão também soropositivos para HCV. Ao exame de admissão: paciente comatoso ( Glasgow = 6 ); pressão arterial 130/80 mmHg; pulso $120 \mathrm{bpm}$; temperatura axilar $40^{\circ}$. Campos pleuro-pulmonares com roncos difusos, precórdio sem alterações, abdome sem massas palpáveis ou alteração de ruídos hidroaéreos; pupilas isocóricas fracamente reagentes a luz, motilidade ocular extrínseca normal, hiperextensão cervical, sem outras alterações de nervos cranianos. Hipertonia muscular generalizada, hiperreflexia generalizada com clônus aquileu inesgotável bilateralmente. Admitido na unidade de terapia intensiva, foram iniciados cuidados de suporte, antitérmico endovenoso, fenitoína $1 \mathrm{~g}$ endovenosa e foi suspensa administração dos neurolépticos. Hemograma: sem alterações na série vermelha, leucocitose com desvio à esquerda ( 17000 leucócitos $/ \mathrm{mm}^{3} ; 67$ segmentados, 7 bastões), plaquetas normais em número e morfologia. Glicemia $110 \mathrm{mg} / \mathrm{dl}$; creatinina $1,2 \mathrm{mg} / \mathrm{dl}$; sódio 139 mEq/l; potássio 3,9 mEq/l; cálcio 9,7 mg/dl; STGP/ALT 62 U/l; STGO/AST 36 U/l; CPK 1890 U/l; LDH 338 U/l. Gasometria arterial, pH 7,526; PO2 49 mmHg; PCO2 25,3 mmHg; BE 0,5 mmol/l; sat. O2 89\% em ar ambiente. Tomografia de crânio sem evidência de lesões expansivas ou hemorragia. A punção lombar evidenciou líquor discretamente amarelado, límpido, com 4,3 leucócitos $/ \mathrm{mm} 3,6,2$ hemácias $/ \mathrm{mm} 3$, proteína $83,7 \mathrm{mg} / \mathrm{dl}$, glicose 71 $\mathrm{mg} / \mathrm{dl}$. Foi firmado diagnóstico de síndrome neuroléptica maligna. O paciente evoluiu com aumento dos níveis séricos de CPK ( CPK $8040 \mathrm{U} / 1$ no $2^{\circ}$ dia de internação). No $2^{\circ}$ dia de internação radiografia de tórax monstrou condensações em ambas as bases pulmonares compatíveis com broncopneumonia sendo iniciado tratamento com ceftriaxone $2 \mathrm{~g}$ EV ao dia e clindamicina $600 \mathrm{mg}$ EV de $8 / 8$ horas. Evoluiu com queda dos níveis séricos de CPK, melhora progressiva do nível de consciência, mantendo-se com diurese normal e creatinina sérica estável durante todo o internamento. Recebeu alta hospitalar no $10^{\circ}$ dia pós admissão em bom estado geral, sem déficits. O paciente foi encaminhado a um serviço psiquiátrico onde reiniciou-se tratamento antipsicótico com olanzapina $5 \mathrm{mg}$ ao dia com aumento progressivo até a dose de $10 \mathrm{mg}$ ao dia.

O paciente retornou ao nosso serviço aproximadamente 2 meses após a alta. A esposa referiu parada de uso de olanzapina 2 dias antes desta admisssão, segundo orientação do psiquiatra. Referiu ainda que no dia desta admissão, o paciente apresentou episódio de perda de consciência com queda ao solo. Ao exame apresentava pulso $114 \mathrm{bpm}$, frequência respiratória $36 \mathrm{ipm}$, pressão arterial 140/80 $\mathrm{mmHg}$; grau 5 na escala de coma de Glasgow, pupilas midriáticas, isocóricas, pouco fotorreagentes e desvio ocular superior. Havia ainda rigidez muscular severa, tremor de extremidades, sudorese profusa, e foi notada liberação esfincteriana durante o exame. Demais passos de exame sem alterações. Hemograma: hemácias 5100 000/ $\mathrm{mm}^{3}$, hemoglobina $15 \mathrm{~g} / \mathrm{dl}$, VG 44\%; leucócitos $21700 / \mathrm{mm}^{3}$ (segmentados $60 \%$, bastões $13 \%$, linfócitos $17 \%$, monócitos $10 \%$ ), plaquetas $330000 / \mathrm{mm}^{3}$. Glicemia $108 \mathrm{mg} / \mathrm{dl}$, sódio $138 \mathrm{mEq} / \mathrm{L}$, potássio $3,7 \mathrm{mEq} / \mathrm{l}$, creatinina $1,0 \mathrm{mg} / \mathrm{dl}$, cálcio 8,6 mg/dl, CPK $324 \mathrm{U} / \mathrm{l}$. Radiografia de tórax normal. Gasometria arterial: pH 7,42, $\mathrm{PCO} 236,4 \mathrm{mmHg}, \mathrm{PO} 252 \mathrm{mmHg}$, sat. O2 87,2\%. Foi admitido à Unidade de Terapia Intensiva e entubado por via orotraqueal. Foram iniciados bromocriptina 2,5 $\mathrm{mg} 2$ vezes ao dia e dantrolene $180 \mathrm{mg}$ ao dia. A punção lombar mostrou líquor límpido sem alterações à análise bioquímica e microbiológica. Dois dias após a admissão ocorreu crise convulsiva manejada com hidantoína. Ainda no segundo dia desta admissão, radiografia de tórax monstrou broncopneumonia bibasal, motivo pelo qual iniciou-se ceftriaxone. No dia seguinte o potássio sérico era $2,3 \mathrm{mEq} / 1$, sendo prontamente iniciada correção. Dois dias após início de ceftriaxone, o quadro pulmonar manteve-se inalterado, sendo mudado o esquema 
antibiótico para ceftazidime associada a clindamicina. Os níveis séricos de CPK atingiram nível de 2504 U/1 no $4^{\circ}$ dia da admissão. Níveis de creatinina sérica permaneceram normais durante todo o internamento. Paciente evoluiu bem do ponto de vista clínico sendo transferido para unidade de tratamento psiquiátrico 13 dias após a admissão.

\section{DISCUSSÃO}

A síndrome neuroléptica maligna é uma reação idiossincrática ao uso de neurolépticos, tendo já sido descrita como ocasionada pela retirada de agentes antiparkinsonianos e drogas depletoras de dopamina.

Não existe definição universalmente aceita para $\mathrm{SNM}^{4-6}$. O diagnóstico baseia-se em critérios clínicos e laboratoriais além da exclusão de outras possíveis causas de quadros semelhantes. Segundo a Associação Americana de Psiquiatria, no DSM-IV ${ }^{7}$, são necessários rigidez muscular severa e febre acompanhado de no mínimo 2 dos 10 itens seguintes: diaforese, disfagia, tremor, incontinência, alteração do estado mental, mutismo, taquicardia, pressão arterial elevada ou lábil, leucocitose, creatinofosfoquinase elevada. Alguns outros autores sugerem critérios mais ou menos rígidos não cabendo tal discussão no presente relato ${ }^{4,8}$.

A incidência de SNM na população varia muito, de acordo com o tipo de estudo realizado (retrospectivo ou prospectivo ) e na dependência dos critérios diagnósticos empregados ${ }^{9}$. Estes números variam de $0,07 \%{ }^{10}$ até $12,2 \%$ segundo Addonizio e col., os quais relatam que caso fossem levados em conta os casos parciais a incidência por eles descrita passaria de $2.4 \%$ para $12,2 \%{ }^{11}$. Keck e col. em estudo prospectivo realizado em 679 pacientes consecutivos tratados com neuroléptico encontraram número próximo a $1 \%{ }^{12-14}$.

O agente causal mais frequente é o haloperidol. Lavie e col. relataram história de uso de haloperidol em 55\% dos 55 casos de SNM estudados. Os mesmos autores citam a clorpromazina como envolvida em $29 \%$ dos $\operatorname{casos}^{15}$. No presente relato, o haloperidol (associado ao cloridrato de clorpromazina ) foi um dos responsáveis pela ocorrência do primeiro episódio da síndrome. Diversas outras drogas têm sido citadas como causadoras de SNM entre elas flufenazina, lítio, tioridazina, clozapina, metoclopramida, tetrabenazine, ecstasy, carbamazepina, entre outras. Também Lavie e cols. relatam um caso de 3 episódios de SNM em um único paciente tratados com três drogas diferentes ${ }^{16}$. No conhecimento dos autores este é primeiro caso da literatura associado ao uso de olanzapina, a qual foi responsável pelo segundo episódio no presente relato.

Condições clínicas associadas como doença de Parkinson ${ }^{17}$ em pacientes sem exposição prévia a neurolépticos e infarto agudo do miocárdio ${ }^{18}$ também encontram-se descritas.

Alguns fatores de risco para o aparecimento desta síndrome têm sido descritos tais como agitação, exaustão física, neuroleptização rápida ${ }^{9,15,19}$. Shalev e col. descrevem a ocorrência de 3 casos de SNM relacionados a uma onda de calor a qual os autores imputam papel coadjuvante no aparecimento da síndrome ${ }^{20}$.

A fisiopatologia da SNM ainda não está definida. Acredita-se que ela se deva a bloqueio dos receptores dopaminérgicos nos gânglios da base, fato este que deu origem à sinonímia de síndrome da deficiência aguda de dopamina ${ }^{3,9,15}$. Tanii e col. descreveram modelo animal baseado em coelhos expostos ao calor após administração de haloperidol e atropina, na tentativa de melhor compreender a etiopatogenia e avaliar os tratamentos disponíveis para síndrome ${ }^{21}$.

As manifestações clínicas da SNM abrangem largo espectro. O quadro mais típico é de alteração do estado mental e rigidez muscular precedendo ou acompanhado de febre ${ }^{22}$. Em revisão de 55 casos de SNM, Lavie e col. encontraram febre em $100 \%$ dos casos, rigidez em $91 \%$, taquicardia em $85 \%$, alteração do estado mental em $76 \%$, entre outras. Os mesmos autores relatam aumento de CPK em $92 \%$ dos casos e leucocitose em $90 \%{ }^{15}$. Adityanjee alerta para o fato da má interpretação de 
dosagem de CPK elevada, ressaltando a possibilidade de ocorrência de tal fato pelo mero uso de neuroléptico via intramuscular ou por quadro de agitação, sendo a elevação desta enzima um fenômeno inespecífico ${ }^{23}$.

No diagnóstico diferencial da SNM várias situações clínicas devem ser consideradas, sendo diagnóstico de exclusão em pacientes com alteração do estado mental, febre e hipertonia. Infecções causando exacerbação de sinais extrapiramidais pré-existentes e diminuição de nível de consciência podem erroneamente ser interpretados como SNM. Pneumonia seguida de reação distônica generaliza é, sem dúvida, o principal diagnóstico diferencial da SNM. Síndrome de abstinência como delirium tremens e interações medicamentosas também devem ser excluídas. Uma síndrome semelhante em pacientes psicóticos não tratados, conhecida como catatonia letal, caracterizada por uma fase prodrômica de mania, anorexia, comportamento destrutivo seguida de catatonia, febre e instabilidade autonômica pode simular $\mathrm{SNM}^{3}$. No caso por nós descrito, os referidos diagnósticos diferenciais form excluídos através de história, exame físico e exames complementares.

Sérias complicações frequentemente se desenvolvem em pacientes com SNM, sendo que mais de um terço destes necessitam de cuidados em unidade de terapia intensiva ${ }^{9,24}$. A maior causa de morbidade e mortalidade é a insuficiência respiratória. Esta pode ser ocasionada por broncoaspiração, taquipnéia superficial com hipoventilação, embolia pulmonar, choque. Outras complicações são a insuficiência renal secundária à rabdomiólise e mioglobinúria e as convulsões ${ }^{3,9,15,20}$.

No tratamento da SNM estão incluídas medidas de suporte como hidratação, suporte ventilatório e nutricional adequados, prevenção de eventos tromboembólicos através do uso de heparina em baixas doses s $^{3,9,15,24-26}$.

O tratamento específico da síndrome permanece controverso ${ }^{3}$. Embora vários estudos relatem o benefício do uso de dantrolene e/ou bromocriptina ${ }^{9,15,25,26}$, lisuride ${ }^{27}$, eletroconvulsoterapia ${ }^{28}$, plasmaferese $^{29}$, levodopa intravenosa ${ }^{30}$, carbidopa/levodopa ${ }^{31}$, ainda existem algumas dúvidas sobre tal valor. Rosenbush e col. ${ }^{32}$ relatam um prolongamento da síndrome quando do uso de bromocriptina associada a dantrolene, porém estes dados foram obtidos em análise retrospectiva. Os pacientes tratados com esta combinação apresentaram alto índice de complicações médicas não relacionadas à SNM e à duração dos sintomas nos pacientes tratados suportivamente foi menor que a média encontrada na literatura ${ }^{32}$. Já Lavie e col. em revisão de 55 casos, descrevem o uso de dantrolene como de grande ajuda em $89 \%$ dos 9 casos em que a droga foi utilizada ${ }^{15}$. Kellam ${ }^{9}$ em metanálise de 536 casos, entre casos próprios e de literatura, cita o uso de dantrolene em 44 pacientes com índice de falha de $16 \%$; para bromocriptina este índice foi de $16 \%$ ( 5/42) e para a associação das duas drogas o índice foi de 14\% (3/22). Provavelmente exista um efeito benéfico marcante no uso destas drogas, porém seu real valor ainda precisa ser confirmado através de estudos adequados.

O prognóstico da SNM depende fundamentalmente da precocidade do diagnóstico e do tratamento instituído ${ }^{33}$. A mortalidade da síndrome varia de 9 a 30\%. Lavie e col. em revisão da literatura inglesa encontraram mortalidade de $9 \%$ atribuindo este menor índice em parte ao diagnóstico precoce da entidade. Kellam relata que o pico de temperatura permanece associado ao da sobrevida; todos os 38 pacientes com temperaturas entre 37,5 e $37,9^{\circ} \mathrm{C}$ sobreviveram; entre 38 e $38,9^{\circ} \mathrm{C}, 6$ pacientes de 92 morreram; entre 39 e $39,9^{\circ} \mathrm{C}, 10$ de 85 morreram; entre 40 e $40,9^{\circ} \mathrm{C}, 18$ de 79 morreram; entre 41 e $41,9^{\circ} \mathrm{C} 17$ de 55 faleceram e finalmente acima de $42^{\circ} \mathrm{C} 14$ pacientes morreram dentre os 25 revisados.

Concluimos que a SNM constitui uma complicação do uso de neurolépticos mais comum do que se imaginava devendo seu diagnóstico e manejo ser do conhecimento não só do psiquiatra e neurologista mas também do clínico geral, fato este que pode levar ao diagnóstico mais precoce da síndrome, o qual contribui decisivamente para o sucesso do tratamento e para o completo restabelecimento dos indivíduos acometidos. 


\section{REFERÊNCIAS}

1. Delay J, Pichot P, Lemperiere T. Un neuroleptique majeur non phenothiazine et non reserpinique l'haloperidol dans le traitment des psychoses. Ann Med Psychol ( Paris )1960;118:145-152.

2. Delay J, Deniker P. Drug-induced extrapyramidal syndromes. In Vinken PJ, Bruyn GW (eds). Diseases of basal ganglia. Handbook of Clinical Neurology Vol 6. Amsterdam: North Holland, 1968: 248-266.

3. Friedman JH. Neuroleptic malignant syndrome. In Gilman S, Goldstein GW, Waxman SG(eds). Neurobase. 3.Ed. San Diego: Arbor, 1977.

4. Gurrera RJ, Chang SS, Romero JA. A comparison of diagnostic criteria for neuroleptic malignant syndrome. J Clin Psychiatry 1992;53:56-62.

5. Buckley PF, Hutchinson M. Neuroleptic malignant syndrome. J Neurol Neurosurg Psychiatry 1995;58:271-273

6. Sternberg DE. Neuroleptic malignant syndrome: the pendulum swings. Am J Psychiatry 1986;143:1273-1275.

7. American Psychiatry Association. Diagnostic and statistical manual for mental disorders. 4.Ed. Washington, DC: American Psychiatry Association, 1994:739-742.

8. Levenson JL. Neuroleptic malignant syndrome. Am J Psychiatry 1985;142:1137-1145.

9. Kellam AMP. The ( frequently ) neuroleptic ( potentially ) malignant syndrome. Br J Psychiatry 1990;157:169-173.

10. Gelenberg AJ, Bellinghausen B, Wojcik JD, Falk WE, Sachs GS. A prospective study of neuroleptic malignant syndrome in a short term psychiatric hospital. Am J Psychiatry 1988;145:517-518.

11. Addonizio G, Susman VL, Roth SD. Symptoms of neuroleptic malignant syndrome in 82 consecutive patients. Am J Psychiatry 1986;143:1587-1590.

12. Keck PE Jr, Pope HG Jr, McElroy SL. Frequency and presentation of neuroleptic malignant syndrome: a prospective study. Am J Psychiatry 1987;144:1344-1346.

13. Keck PE Jr, Pope HG Jr, McElroy SL. Declining frequency of neuroleptic malignant syndrome in a hospital population . Am J Psychiatry 1991;148:880-882.

14. Pope HG Jr, Keck PE Jr, McElroy SL. Frequency and presentation of neuroleptic malignant syndrome in a large psychiatric hospital. Am J Psychiatry 1986;143:1227-1233.

15. Lavie CJ, Olmsted TR, Ventura HO, Lepler BJ. Neuroleptic malignant syndrome: an underdiagnosed reaction to neuroleptic agents? Postgraduate Med 1986;80:171-178.

16. Lavie CJ, Ventura HO, Walker G. Neuroleptic malignant syndrome: three episodes with different drugs. Southern Med J 1986;79:1571-1573.

17. Keyser DL, Rodnitzky RL. Neuroleptic malignant syndrome in Parkinson's diesease after withdrawal or alteration of dopaminergic therapy. Arch Intern Med. 1991;151:794-796.

18. Freedman MD. Neuroleptic malignant syndrome and acute myocardial infarction: case report and review. Md Med J. 1997;46:357-359.

19. Pope HG Jr, Keck PE Jr, Cohen BM, Nierenberg AA. Risk Factors for neuroleptic malignant syndrome: a case-control study. Arch Gen Psychiatry 1989;46:914-918.

20. Shalev A, Hermesh H, Munita $\mathrm{H}$. The role of external heat load in triggering the neuroleptic malignant syndrome. Am J Psychiatry 1988;145:110-111.

21. Tanii H, Taniguchi N, Niigawa H, et al. Development of an animal model for neuroleptic malignant syndrome: heatexposed rabbits with haloperidol and atropine administration exhibit increased muscle activity, hyperthermia and serum creatine phosphokinase level. Brain Res 1996;16:1-2,263-270.

22. Rosebush PI, Stewart T. A prospective analysis of 24 episodes of neuroleptic malignant syndrome. Am J Psychiatry 1989;146:717-725.

23. Adityanjee. The myth of elevated serum creatine phosphokinase level and neuroleptic malignant syndrome. Br J Psychiatry 1991;158:706-707.

24. Montgomery JN, Ironside JW. Neuroleptic malignant syndrome in the intensive therapy unit. Anaesthesia 1990;45:311-313.

25. Janati A, Webb T. Successful treatment of neuroleptic malignant syndrome with bromocriptine. Southern Med J 1986;79:1567-1571.

26. Rosenberg MR, Green M. Neuroleptic malignant syndrome: review of response to therapy. Arch Intern Med 1989;149:1927-1931.

27. Sczesni B, Brittkau S, von Baumgarten Fv, von Schroder J, Suchy I, Przuntek H. Intravenous lisuride in the treatment of neuroleptic malignant syndrome. J Clin Psychopharmacol 1991;11:185-188.

28. Addonizio G, Susman VL. ECT as a treatment alternative for patients with symptoms of neuroleptic malignant syndrome. J Clin Psychiatry 1987;48:102-105.

29. Gaitini L, Fradis M, Vaida S, Krimerman S, Beny A. Plasmapheresis in neuroleptic malignant syndrome. Anaesthesia 1997;52:165-168.

30. Nisijima K, Noguti M, Ishiguro T. Intravenous injection of levodopa is more effective than dantrolene as therapy for neuroleptic malignant syndrome.Biol Psychiatry 1997;41:913-914.

31. Shoop SA, Cernek PK. Carbidopa/levodopa in the treatment of neuroleptic malignant syndrome. Ann Pharmacother 1997;31:119.

32. Rosebush PI, Stewart T, Mazurek MF. The treatment of neuroleptic malignant syndrome. are dantrolene and bromocriptine useful adjuncts to supportive care? Br J Psychiatry 1991;159:709-712.

33. Pope HG Jr, Aizley HG, Keck PE Jr, McElroy SL. Neuroleptic malignant syndrome: long-term follow-up of 20 cases. J Clin Psychiatry 1991;52:208-212. 